\title{
Unity and diversity of executive functions in creativity
}

Darya L. Zabelina ${ }^{1}$, Naomi P. Friedman ${ }^{2}$, Jessica Andrews-Hanna ${ }^{3}$

\author{
${ }^{1}$ University of Arkansas \\ ${ }^{2}$ University of Colorado Boulder \\ ${ }^{3}$ University of Arizona
}

This research was supported in part by the Imagination Institute's grant from the Templeton Foundation, RFP-15-04 to DLZ, and grant MH063207 to NPF.

Correspondence concerning this article should be addressed to Darya L. Zabelina, Department of Psychological Science, 480 Campus Drive, Fayetteville, AR 72701. Ph:479/575-5813. E:

dlzabeli@uark.edu. 


\begin{abstract}
Increasing evidence suggests that executive functions (EFs) - a set of general-purpose control processes that regulate thoughts and behaviors - are relevant for creativity. However, EF is not a unitary process, and it remains unclear which specific EFs are involved. The present study examined the association between the three EFs, both uniquely (EF-Specific) and together (Common EF), and three measures of creativity. Participants $(\mathrm{N}=47)$ completed a divergent thinking test, and self-reported their real-life creative accomplishments. A subset of participants indicated their involvement in the artistic or information technology (IT) professions. Results indicated that fluency (but not originality) of divergent thinking was uniquely predicted by working memory Updating. Better response Inhibition predicted higher number of real-world artistic creative achievements. Involvement in the artistic (versus IT) professions was associated with better Common EF, and with enhanced mental set Shifting abilities. Results demonstrate that different EFs predict creativity depending on its operational definition.
\end{abstract}

Keywords: Executive control; creativity; intelligence; divergent thinking 


\section{Unity and diversity of executive functions in creativity}

\section{Introduction}

Increasing evidence suggests that executive functions (EFs) - a set of general-purpose control processes that regulate one's thoughts and behaviors (Friedman \& Miyake, 2017) - are relevant for creativity; however, it is not clear which specific EFs are involved. Commonly postulated EFs include Updating, Shifting, and Inhibition (Miyake \& Friedman, 2012; Miyake, Friedman, Emerson, Witzki, \& Howerter, \& Wager, 2000). Updating refers to the ability to monitor and rapidly add or delete the contents of working memory; Shifting refers to the ability to flexibly shift between different tasks or mental sets; and Inhibition refers to the ability to purposefully suppress or override dominant, but irrelevant response tendencies. Updating, the EF most closely linked with intelligence (Ackerman, Beier, \& Boyle, 2005; Friedman, Miyake, Corley, Young, DeFries, \& Hewitt, 2006), has been shown to relate to creative cognition (e.g., Batey \& Furnham, 2006; Kim, Cramond, \& VanTassel-Baska, 2010; Nusbaum \& Silvia, 2011). Response inhibition has been linked with ideational fluency, i.e., the ability to rapidly produce a series of ideas (Benedek, Franz, Heen, \& Neubauer, 2012), and with fluency and flexibility, but not with originality on a problem-finding task (Cheng, Hu, Jia, \& Runco, 2016). The contribution of Shifting ability to creativity, however, is less clear.

Creativity, commonly defined as the ability to produce novel and useful responses (Runco \& Jaeger, 2012), may indeed be characterized by all three EFs. Novelty of creative thinking, for instance, may be reflected in the ability to inhibit or avoid common paths in service of a novel or unique idea. The ability to monitor and add or delete contents of working memory may be beneficial for considering and recombining unrelated concepts. And overriding dominant responses and flexibly shifting to more original ideas may also contribute to creative cognition. Hence, the present study aims to examine the specific and common contribution of Updating, Shifting, and Inhibition to creativity.

The unity/diversity model of EFs (Miyake et al., 2000) suggests that the three EFs are correlated, thus tapping into a common underlying ability (unity). However, latent models also reveal separability 
(diversity) across EFs, suggesting that there is variance unique to particular EF. Several independent studies have shown that after accounting for Common EF, only Updating and Shifting exhibit unique variance, while Inhibition is entirely explained by the Common EF factor (Fleming, Heintzelman, \& Bartholow, 2016; Friedman, Miyake, Young, Defries, Corley, \& Hewitt, 2008; Ito et al, 2015).

Interestingly, EFs sometimes show opposing patterns of correlations (Friedman \& Miyake, 2017; Miyake \& Friedman, 2012). For example, Common EF and Updating-specific abilities relate positively to intelligence, whereas Shifting-specific abilities relate negatively to intelligence (Friedman et al., 2011). Further, children with worse self-restraint show better Shifting-specific abilities in late adolescence (Friedman et al., 2011). Notably, worse self-restraint has been previously linked with increased creativity (Healey \& Rucklidge, 2008). These EFs tend to be highly stable, with substantial correlations at the level of latent variables: .82 for Common EF, 1.00 for Updating-specific ability, and .93 for Shifting-specific ability between the ages of 17 and 23 (Friedman, Miyake, Altamirano, Corley, Young, Rhea, \& Hewitt, 2016).

Despite hypotheses regarding relationships between creativity and EFs, no prior studies have examined both the unity and diversity of EFs in creativity. A number of investigations, however, have reported on the link between creativity and working memory updating, set shifting, and response inhibition, separately, with contradicting results. Several studies suggested that creativity is linked with better response inhibition (Edl, Benedek, Papousek, Weiss, \& Fink, 2014; Groborz \& Necka, 2003), as well as with an elevated ability to avoid repetitive responses (Benedek et al., 2012; Zabelina, Robinson, Council, \& Bresin, 2012). Some even argue that creativity might be not so different from intelligence (Nusbaum \& Silvia, 2011). Creativity has been found to correlate with working memory (de Dreu, Nijstad, Baas, Wolsink, \& Roskes, 2012), and with flexible cognitive control (Zabelina \& Robinson, 2010). Behavioral evidence for the role of EFs in creativity is supported by the electroencephalogram (EEG) and functional magnetic resonance imaging (fMRI) research, showing task-related activation in brain regions associated with interference resolution, response selection, and cognitive control in the 
inferior frontal gyrus (IFG) and inferior parietal cortex (Abraham, Beudt, Ott, \& von Cramon, 2012; Benedek, Jauk, Fink, Koschutnig, Reishofer, Ebner, \& Neubauer, 2014; Chrysikou \& Thompson- Schill, 2011; Fink et al., 2009).

Opposing evidence comes from studies showing that creativity may be characterized by, and in fact benefit from, the lack of cognitive control (Eysenck, 1995; Martindale, 1999; Thomson-Schill, Ramscar, \& Chrysikou, 2009), decreased filtering of task-irrelevant information (Carson, Peterson, \& Higgins, 2003), as well as "leaky" or diffused attention (Zabelina, Saporta, \& Beeman, 2016), and "leaky" sensory processing, as assessed by the P50 ERP (Zabelina, O’Leary, Pornpattananangkul, Nusslock, \& Beeman, 2015). Neuroimaging evidence provides partial support for these findings. For example, spontaneous activity in a set of midline and inferior parietal regions that activate during internallyoriented cognition (i.e., the default mode network; Andrews-Hanna, Reidler, Sepulcre, Poulin, \& Buckner, 2010; Buckner, Andrews-Hanna, \& Schacter, 2008), is associated with the initial stages of the creative process, such as idea generations (Ellamil, Dobson, Beeman, \& Christoff, 2011), and composition of poetry (Liu, Erkkinen, Healey, Xu, Swett, Chow, \& Braun, 2015). Idea revision and poetry evaluation involve both the default mode network and the frontoparietal control network activity (for a review, see Zabelina \& Andrews-Hanna, 2016). The dynamic framework model has also suggested that creativity may involve rapid switching between controlled and spontaneous processing (Christoff, Irving, Fox, Spreng, \& Andrews-Hanna, 2016).

A key question when examining prior literature on the role of EFs in creativity is the operational definition of creativity. The majority of studies assess creativity through performance on divergent thinking tests, such as a version of the Alternate Uses Task (Wallach \& Kogan, 1965), or the Torrance Test of Creative Thinking (Goff \& Torrance, 2002; Torrance, 1974). Divergent thinking tests typically require participants to generate multiple uses for a common object, such as a brick, within a limited amount of time (3-10 mins) in a laboratory setting. Responses are scored for fluency (the number of responses generated within the allotted time), and originality (the statistical uniqueness of each response). 
Recent studies have also used raters' evaluations of a single holistic judgment of “creativity" (e.g., Silvia et al., 2008).

To our knowledge, the only study to directly examine the role of Updating, Shifting, and Inhibition in performance on a divergent thinking task found that people with successful performance on divergent thinking tests also scored better on measures of Updating and Inhibition (Benedek, Jauk, Sommer, Arendasy, \& Neubauer, 2014). These results show that the ability to monitor and rapidly add or delete the contents of working memory, as well as the ability to purposefully suppress prepotent response tendencies in service of novel ideas, may be the core mechanisms for divergent thinking. It should be noted, however, that in Benedek and colleagues' (2014) investigation, divergent thinking was operationalized with a holistic “creativity" score. Furthermore, Updating, Shifting, and Inhibition were examined as separate EFs, with a single task per each EF, which were virtually unrelated (possibly because only one task per EF was used). The contribution of Common EF was also not assessed. The first goal of the present study, therefore, was to expand on these findings, and examine how two components of divergent thinking, namely fluency and originality, relate to both the unity (Common EF), and diversity of EFs, measured with two tasks per EF.

The second goal was to examine the role of EFs in real-life creativity. Prior evidence suggests that, unlike divergent thinking, self-reported creative accomplishments are linked with "leaky" or diffused attention (Zabelina et al., 2016), and with low latent inhibition (Carson et al., 2003). Indeed, diffused attention, as well as low EFs, may help creative people notice information other people may not notice, leading to the generation of creative ideas. However, research also shows that more (compare to less) creative people are better able to maintain focus for longer periods of time. For example, artists (compared to non-artists) spend more time re-working their drawings (Kozbelt, 2008), and design students (compared to non-design students) show better cognitive control, as measured by inhibition abilities on the Stroop task (Edl et al., 2014). Arguably, persistence in the discovery process does not come easy, and while Shifting-specific abilities may help people make connections between distantly 
related ideas, overall high EFs may help creative people guide their cognition in most appropriate ways. In other words, although flexibility and stability have been described in terms of a trade-off (Friedman \& Miyake, 2017; Goeschke, 2000; Herd, O’Reilly, Hazy, Chatham, Brant, \& Friedman, 2014; Miyake \& Friedman, 2012), where the ability to actively maintain a single task goal may be a force that makes it difficult for some people to flexibly switch to a different goal, it is possible that creative people have the propensity towards both.

Indeed, the Model of Creativity and Attention (MOCA; Zabelina, 2018) proposes that real-life creativity may be a product of bottom-up "leaky" or diffused attention, as well as enhanced top-down cognitive control. In other words, what may define creative people is their propensity for flexible shifting between goals or ideas, as well as the ability to regulate their thoughts and behaviors, perhaps to sustain attention toward an overarching goal, and hone in on successful ideas. Thus, according to MOCA, successful performance on laboratory tests of divergent thinking partially depends on high overall cognitive control (i.e., enhanced EF), as well as enhanced Shifting-specific abilities.

\subsection{Aims of the study}

The aim of the present study was thus to examine how specific EFs (Updating, Shifting, and Inhibition), uniquely and in combination (Common EF), relate to real-life creativity and to the two components of divergent thinking, namely fluency and originality. Real-life creativity was assessed with a validated self-reported measure of creative accomplishments (the Creative Achievement Questionnaire; Carson, Peterson, \& Higgins, 2005), and with people's professional involvement in either information technology (IT) or artistic professional domains. Although creativity may be equally important in both professions, artistic professions, such as dancing, creative writing, and photography are traditionally thought of as requiring more creativity compared to those of computer engineering and software implementation professions (e.g., Feist, 1998). Thus, an overarching purpose of the investigation was to examine whether and how Updating, Shifting, and Inhibition, together and uniquely, predict creativity in the real life versus creativity in a laboratory setting. 
All participants completed a laboratory measure of divergent thinking, the Creative Achievement Questionnaire, and if working, indicated whether they are involved in the IT or artistic professional domains. Participants also completed six EF tasks, comprised of the two highest-loading tasks per each EF in the unity/diversity model (Friedman et al., 2016; Ito et al., 2015), and the general cognitive abilities task, which was used as a control measure, as creativity and intelligence may be correlated (Jauk, Benedek, Dunst, \& Neubauer, 2013; Silvia, 2008). Based on prior work (Benedek et al., 2014; De Dreu et al., 2012), we expected that the number of responses people generate on the divergent thinking test (fluency), as well as the uniqueness of responses (originality) would be associated with both working memory updating (Updating) and response inhibition (Inhibition). Further, based on the MoCA (Zabelina, 2018), we predicted that people in the artistic (compared to the IT) professional domains would exhibit enhanced Common EF, as well as enhanced set shifting (Shifting) abilities.

\section{Material and method}

\subsection{Participants and design}

Participants were 47 people (23 male, 24 female, mean age $=29.26, S D=7.93$, range $=19-47$, 98\% White) recruited via Craigslist.com website to participate in the study. Participants responded to an advertisement placed under the 'computer gigs' or the 'creative gigs' sections, and received $\$ 90$ for participation (as part of a larger study). Participants were fluent in English, right-handed, and had no history of brain damage. The study was approved by the local Institutional Review Board.

\section{Materials}

2.2 Abbreviated Torrance Test for Adults (ATTA; Goff \&Torrance, 2002)

To assess divergent thinking, participants completed the ATTA - a shortened form of the Torrance Test of Creative Thinking (Torrance, 1974). The ATTA consists of three activities (3 minutes each), one involving verbal (written) responses (e.g., generating problems that may arise from being able to walk on air or fly without being in an airplane or a similar vehicle), and two involving figural responses (e.g., using incomplete figures to make pictures). Responses were scored for fluency (i.e., the number of 
pertinent responses), and originality (i.e., the number of responses that are not typically produced, according to normative data), with scores summed across the three activities (Goff \& Torrance, 2002). One participant did not complete the ATTA due to experimenter error.

\subsection{Creative Achievement Questionnaire (CAQ; Carson et al., 2005)}

Real-world creative behavior was assessed with the Creative Achievement Questionnaire, in which participants catalogued their prior creative achievements across ten creative domains (visual art, music, dance, architectural design, creative writing, humor, inventions, scientific discovery, theater and film, and culinary arts). In the music domain, for example, questions range from "I have no training or recognized talent in this area" (score of 0) to "my compositions have been critiqued in a national publication" (score of 7). In the scientific discovery subset, scores vary from "I have no training or recognized ability in this field" (score of 0) to "my work has been cited by other scientists in national publications" (score of 7). Separate domain scores were then combined to form a single index of artistic $(M=15.13, S D=14.53$, range $=0-61)$, scientific $(M=9.17, S D=14.96$, range $=0-72)$, and total $(M=$ 24.30, $\mathrm{SD}=21.40$, range $=3-108)$ creative achievements. CAQ scores were positively skewed, and so we used the log transformation to normalize the CAQ distributions.

\subsection{IT and Artistic professional occupations}

A subset of participants (those who were working) indicated their occupation, and were categorized as involved in the "IT" or the "artistic" professional domains. Sixteen participants' professions were classified as IT (software implementation, software engineer, product manager at an information technology company). Nineteen participants' professions were classified as artistic (e.g., artist, writer, composer, musician, photographer, theatrical performer). Fifteen participants indicated their profession as "student" or "unemployed," and were not considered in the analysis specific to the professional occupations, but were included in all other analyses. IT and Artistic groups did not differ in gender (IT: 9 male, 7 female; artistic: 9 male, 10 female; $\chi^{2}(1)=.28, p=.60$ ), nor did they differ in age, 
level of education, or general cognitive abilities as measured by the ICAR test (Condon \& Revellle, 2014; see Table 1).

2.5 The International Cognitive Ability Resource (ICAR; Condon \& Revelle, 2014)

The ICAR assessed general cognitive abilities. The measure contains 16 items assessing letter and number series, matrix reasoning, three-dimensional rotation, and verbal reasoning. Participants were given 18 minutes to complete as many items as they could within the allotted time. The dependent measure was the number of correctly solved items $(M=9.17, S D=3.57$, range $=2-15)$.

\subsection{Executive Function (EF) tasks}

The EF battery comprised six measures of commonly studied EFs: response inhibition, working memory updating, and set shifting, which were programmed in ePrime and administered on a PC. Here the tasks and the task requirements are briefly discussed; for full task details and scoring procedures see Friedman et al. (2016).

The two Inhibition tasks required participants to stop a dominant or prepotent response (reflexive eye movements for Antisaccade, word reading for Stroop). Dependent measures were proportion correct for Antisaccade (reverse-scored), and response time interference (to name colors of incongruent vs. congruent words) for Stroop. The two Updating tasks required participants to continuously update the contents of working memory (with category exemplars for keep track, and letter series for letter memory). Dependent measures were recall accuracy for Keep Track and rehearsal accuracy for Letter Memory. The two Shifting tasks required participants to switch between categorization dimensions (odd/even vs. vowel/consonant for Number-Letter, and big/small vs. living/nonliving for Category Switch), according to a cue stimulus. Dependent measures were local switch costs, average RT on trials that required a switch of categorization rule from the immediately prior trial minus average RT on trials that required a repeat of the same categorization rule (in "mixed" blocks of trials in which both rules were used). All dependent measures were z-scored, and the two tasks within each EF were averaged for the Updating, Shifting, and Inhibition scores. The overall EF score was computed by averaging the z-scored Inhibition (Stroop 
reverse-scored), Updating, and Shifting (reverse-scored) scores, with higher scores reflecting better EF abilities.

For the Number-Letter and Category Switch tasks, only trials (within-subjects) with RTs within the 2.5 standard deviations $(S D s)$ from the overall mean were analyzed. Only correct trials were included in the analyses, except for the Stroop task, where accuracy was not recorded due to a programming error ${ }^{1}$. One participant's Stroop effect and switching cost for the Category Switch task were above 2.5 SDs from the overall mean, and were excluded from the analyses.

\subsection{Education Level}

Participants provided information about their level of education on the following scale: $1=$ less than high school, 2 = high school graduate, $3=$ some college, $4=2$-year degree, $5=4$-year degree, $5=$ professional degree, 6 = Doctorate degree.

\subsection{Analytical Strategy}

First, zero-order correlations were performed to examine the association between the six measures used to tap the three EFs, and the association between other variables of interest: ATTA fluency, ATTA originality, creative achievements in the arts, science, total creative achievements, and cognitive abilities. Next, two logistic linear regressions were conducted to examine (1) the effect of overall EF, and (2) the effect of Updating, Shifting, and Inhibition on IT and artistic professional domains. Simple linear regressions were then carried out to predict self-reported artistic, scientific, and total creative achievements. Finally, simple linear regressions were conducted to determine whether overall EF, or unique variance associated with Updating, Shifting, and Inhibition predicted fluency or originality of divergent thinking. All analyses were repeated controlling for general cognitive ability, education level, age, and gender.

\footnotetext{
${ }^{1}$ Although accuracy was not recorded, our prior studies with this task (e.g., Friedman et al., 2016) indicate high accuracy even in the incongruent condition (97\%). Although it is not ideal to include RTs for error trils in the calculations, there remained a positive interference cost $(t(44)=9.48, p<.001$; though smaller in our prior studies). Importantly, it robustly correlated with other Inhibition task (antisaccade, $r=.34$ ), and its correlations with the other measures were within the range of those reported in other studies (e.g., Friedman et al., 2016).
} 


\section{Results}

Descriptive statistics for the six measures used to tap the three EFs (Updating, Shifting, and Inhibition) are presented in Table 2. As sometimes reported for EF tasks (Miyake et al., 2000; Rabbitt, 1997), reliability estimate for the Keep Track task was somewhat low. However, the use of two tasks per construct helps alleviate such problems (Miyake, Emerson, \& Friedman, 2000). Participants made errors on $3 \%$ of trials $(S D=.03)$ on the number letter task, and on $3 \%(S D=.02)$ on the category-switch task.

Zero-order correlations among the six measures, provided in Table 3, were consistent with the results from previous studies on individual differences in EFs (Miyake et al., 1999). Importantly, measures considered to tap the same EF showed significant correlations with one another.

Zero-order correlations among Updating, Shifting, and Inhibition composites showed that the three EFs were significantly correlated with one another (Table 4), consistent with prior work (Friedman et al., 2008). Based on the standard $p$-value statistical significance, fluency and originality of divergent thinking were not associated with any of the EFs, nor was performance on the divergent thinking test associated with real-life creative achievement. However, small to medium effect sizes were found for the associations between the Overall EF and creative achievements in the arts $(r=.19)$, and the science domains $(r=-.16)$. Small to medium effect sizes were found for the associations between Updating ability and the fluency of divergent thinking $(r=.18)$ and cognitive abilities $(r=.19)$. Small to medium effect sizes were also found for the associations between Shifting ability and the fluency of divergent thinking ( $r$ $=-.12)$, creative achievements in the arts $(r=.15)$, and the science domains $(r=-.25)$, and cognitive abilities $(r=-.20)$. Finally, small to medium effect sizes were found for the associations between Inhibition ability and the fluency of divergent thinking $(r=-.14)$, creative achievements in the science domains $(r=-.16)$, and total creative achievements $(r=.22)$, indicating practical significance for the associations between these variables (Cohen, 1992). Higher creative achievements in the arts was associated with better Inhibition ability, $r=.37, p=.01$, with a medium effect size. 
People in the IT and Artistic professional domains did not differ in their performance on the divergent thinking test, nor did they differ in their responses on the self-reported measure of creative achievements (Table 1). Cohen's $d$ effect sizes, however, suggested a small to moderate effect for the two professional domains for the fluency of divergent thinking (IT > Artistic), creative achievements in the arts domains (IT $<$ Artistic), and total creative achievements (IT $<$ Artistic).

\section{Table 1}

Descriptive statistics and independent group t-tests between IT and artistic professions.

\begin{tabular}{|c|c|c|c|c|c|c|c|c|}
\hline & & IT & & Artis & & & & \\
\hline & $M$ & $S D$ & $M$ & $S D$ & $t$-test & $p$ & Cohen's $d$ & $95 \% \mathrm{CI}$ \\
\hline ATTA fluency & 18.00 & 4.84 & 16.22 & 4.63 & 1.09 & .28 & .38 & {$[-1.54 ; 5.09]$} \\
\hline ATTA originality & 9.75 & 5.07 & 9.61 & 3.52 & 0.09 & .93 & .03 & {$[-2.88 ; 3.16]$} \\
\hline CAQ arts & 0.94 & 0.33 & 1.14 & 0.47 & -1.48 & .15 & .49 & {$[-.49 ; .08]$} \\
\hline CAQ science & 0.78 & 0.45 & 0.71 & 0.43 & 0.48 & .63 & .16 & {$[-.23 ; .37]$} \\
\hline CAQ total & 1.15 & 0.38 & 1.34 & 0.35 & -1.51 & .13 & .52 & {$[-.44 ; .06]$} \\
\hline ICAR & 9.31 & 3.28 & 9.37 & 3.58 & -0.05 & .96 & .02 & {$[-2.44 ; 2.32]$} \\
\hline Education level & 4.36 & 1.34 & 4.68 & 1.20 & -0.74 & .47 & .25 & {$[-1.23 ; .58]$} \\
\hline Age & 33.00 & 8.42 & 29.79 & 7.59 & 1.19 & .24 & .40 & {$[-2.30 ; 8.72]$} \\
\hline
\end{tabular}




\section{Table 2}

Descriptive statistics for the measures used to tap Updating, Shifting, and Inhibition.

\begin{tabular}{lllccc}
\hline Task & $M(S D)$ & Range & Skewness & Kurtosis & Reliability \\
& & & & & \\
Letter Memory & $99(22)$ & 56 to 131 & -.15 & -1.13 & $.94^{a}$ \\
Keep Track & $41(4)$ & 31 to 50 & -0.31 & -0.51 & $.48^{a}$ \\
Number-Letter & $200 \mathrm{~ms}(136)$ & -106 to 543 & 0.79 & 0.91 & $.98^{b}$ \\
Category Switch & $204 \mathrm{~ms}(90)$ & 32 to 408 & 0.36 & 0.07 & $.97^{b}$ \\
Stroop & $73 \mathrm{~ms}(52)$ & -16 to 181 & 0.39 & -0.65 & $.99^{b}$ \\
Antisaccade & $79 \%(14)$ & $47 \%$ to $98 \%$ & -0.64 & -0.46 & $.93^{b}$ \\
\hline
\end{tabular}

$\bar{a}$ Reliability was calculated using Cronbach's alpha.

${ }^{b}$ Reliability was calculated by adjusting split-half (odd-even) correlations with the Spearman-Brown prophecy formula. 
Table 3

Pearson correlation coefficients for the six executive function measures.

\begin{tabular}{|c|c|c|c|c|c|c|}
\hline & 1 & 2 & 3 & 4 & 5 & 6 \\
\hline 1. Letter Memory & -- & $.47 * *$ & .10 & $.29^{\dagger}$ & -.11 & $.33^{*}$ \\
\hline 2. Keep Track & & -- & $.57 * *$ & $.56 * *$ & .15 & $.49 * *$ \\
\hline 3. Number-Letter & & & -- & $.46^{* *}$ & .18 & $.36 * *$ \\
\hline 4. Category Switch & & & & -- & $.29^{\dagger}$ & $.55^{*}$ \\
\hline 5. Stroop & & & & & -- & $.34 *$ \\
\hline 6. Antisaccade & & & & & & -- \\
\hline
\end{tabular}

Note. For all tasks, higher values indicate better performance.

$\dagger p<.10 ; * p<.05 ; * * p<.01$. 


\section{Table 4}

Pearson correlation coefficients for the EF, creativity, and cognitive abilities measures.

\begin{tabular}{|c|c|c|c|c|c|c|c|c|c|c|}
\hline & 1 & 2 & 3 & 4 & 5 & 6 & 7 & 8 & 9 & 10 \\
\hline 1. Overall EF & --- & $.76 * *$ & $.86^{* *}$ & $.75^{* *}$ & -.04 & -.02 & .19 & -.16 & .06 & -.04 \\
\hline 2. Updating & & -- & $.52 * *$ & $.32 *$ & .18 & -.01 & .04 & .02 & -.01 & .19 \\
\hline 3. Shifting & & & -- & $.49 * *$ & -.12 & -.07 & .15 & $-.25^{\dagger}$ & .05 & -.20 \\
\hline 4. Inhibition & & & & -- & -.14 & .03 & $.35 *$ & -.16 & .12 & -.08 \\
\hline 5. ATTA fluency & & & & & -- & -.03 & -.11 & -.17 & -.22 & -.01 \\
\hline 6. ATTA originality & & & & & & -- & .15 & .10 & .22 & .05 \\
\hline 7. CAQ arts & & & & & & & -- & .01 & $.73 * *$ & .16 \\
\hline 8. CAQ science & & & & & & & & -- & $.61 * *$ & .19 \\
\hline 9. CAQ total & & & & & & & & & -- & .12 \\
\hline 10. ICAR & & & & & & & & & & -- \\
\hline
\end{tabular}

Note. $\mathrm{EF}=$ Executive Functions; ATTA = Abbreviated Torrance Test for Adults; CAQ = Creative Achievement Questionnaire; ICAR = International Cognitive Ability Resource.

$+p<.10 ; * p<.05 ; * * p<.01$.

\subsection{Divergent thinking and EFS}

Zero-order correlations between fluency and originality of divergent thinking and the EF factors are reported in Table 4. Controlling for general cognitive ability, education level, age, and gender, overall EF did not significantly predict either fluency $(\beta=.06, S E=1.17, t=.34, p=.74)$, or originality $(\beta=.06$, $S E=.95, t=.36, p=.72)$ of divergent thinking. 
Multiple regression analysis was carried out to examine if Updating, Shifting, and Inhibition significantly predicted participants' fluency of divergent thinking. The results of the regression indicted that the three predictors explained $11 \%$ of variance in the fluency of divergent thinking, $\mathrm{R}^{2}=.11, F(3,45)$ $=1.76, p=.17$. Better Updating abilities predicted higher fluency of divergent thinking $(\beta=.35, S E=.94$, $t=2.04, p=.048)$. Further multiple regression examined if Updating, Shifting, and Inhibition predicted participants' fluency of divergent thinking, while controlling for general cognitive ability, education level, age, and gender. The results of the regression indicted that the seven predictors explained $21 \%$ of variance in the fluency of divergent thinking, $\mathrm{R}^{2}=.21, F(7,42)=1.37, p=.25$. Controlling for these other predictors, better Updating abilities significantly predicted higher fluency of divergent thinking $(\beta=.47$, $S E=1.07, t=2.41, p=.02)$, indicating that Updating abilities are linked with the ability to produce a higher number of responses on the divergent thinking task (Table 6). Originality of divergent thinking was not predicted by unique variance associated with Inhibition, Updating, or Shifting, with or without controlling for general cognitive ability, age, and gender, $p \mathrm{~s}>.32$. 


\section{Table 6}

Linear multiple regressions predicting fluency and originality of divergent thinking.

B $\quad \mathrm{SE} \quad \beta \quad t \quad p \quad 95 \% \mathrm{CI}$

DV: ATTA Fluency

$\begin{array}{lcccccc}\text { Updating } & 2.59 & 1.07 & 0.47 & 2.41 * & .02 & {[.41 ; 4.77]} \\ \text { Shifting } & -1.49 & 1.14 & -0.29 & -1.31 & .20 & {[-3.80 ; .81]} \\ \text { Inhibition } & -.93 & 1.08 & -0.16 & -.86 & .40 & {[-3.13 ; 1.27]} \\ \text { ICAR } & -0.21 & 0.22 & -0.17 & -.98 & .33 & {[-.65 ; .23]} \\ \text { Education level } & 0.43 & 0.68 & 0.10 & 0.64 & .52 & {[-.94 ; 1.82]} \\ \text { Age } & 0.05 & 0.11 & 0.09 & 0.48 & .64 & {[-.17 ; .27]} \\ \text { Gender } & 1.53 & 1.50 & 0.16 & 1.02 & .32 & {[-1.52 ; 4.58]}\end{array}$

DV: ATTA Originality

$\begin{array}{lcccccc}\text { Updating } & -0.41 & 0.93 & -0.09 & -.44 & .66 & {[-2.30 ; 1.48]} \\ \text { Shifting } & 0-.04 & 0.98 & -.01 & -.04 & .97 & {[-2.04 ; 1.94]} \\ \text { Inhibition } & .94 & 0.94 & 0.20 & 1.01 & .32 & {[-.96 ; 2.84]} \\ \text { ICAR } & 0.13 & 0.19 & 0.12 & 0.68 & .50 & {[-.25 ; .51]} \\ \text { Education level } & 0.07 & 0.59 & 0.02 & 0.12 & .90 & {[-1.12 ; 1.26]} \\ \text { Age } & 0.09 & 0.09 & 0.19 & .97 & .34 & {[-.10 ; .28]} \\ \text { Gender } & 2.92 & 1.30 & 0.38 & 2.24 * & .03 & {[.28 ; 5.56]}\end{array}$

Note. $\quad$ ATTA = Abbreviated Torrance Test for Adults; ICAR = International Cognitive Ability Resource. 


\subsection{Self-reported creative achievement and EFs}

Zero-order correlations between artistic and scientific creative achievements and EF scores are reported in Table 4. Multiple regressions were used to predict self-reported artistic, scientific, and total creative achievements. Controlling for general cognitive ability, education level, age, and gender, overall EF did not predict overall creative achievements, nor creative achievements in the arts or the science domains, $p \mathrm{~s}>.38$.

Multiple regressions examined whether Updating, Shifting, and Inhibition significantly predicted participants' artistic and scientific creative achievements. The three predictors explained $15 \%$ of the variance in artistic creative achievements, $\mathrm{R}^{2}=.15, F(3,45)=2.52, p=.07$. Controlling for the other EFs, better Inhibition abilities significantly predicted artistic creative achievements $(\beta=.39, \mathrm{SE}=.08, t=2.35$, $p=.02$ ). The three predictors explained $10 \%$ of the variance in scientific creative achievements, $\mathrm{R}^{2}=.10$, $F(3,45)=1.54, p=.22$. Controlling for the other EFs, worse Shifting abilities marginally predicted scientific creative achievements $(\beta=-0.33, S E=.09, t=-1.78, p=.08)$. None of the EF abilities predicted total creative achievements, $p \mathrm{~s}>.48$.

Further a multiple regression analysis was carried out to test if Updating, Shifting, and Inhibition significantly predicted participants' artistic creative achievements, while controlling for general cognitive ability, education level, age, and gender. The results of the regression indicated that the seven predictors explained $28 \%$ of the variance in artistic creative achievements, $\mathrm{R}^{2}=.28, F(7,42)=1.93, p=.09$. Better Inhibition abilities remained marginally significant predictors of artistic creative achievements $(\beta=0.38$, $S E=.09, t=2.02, p=.051)$. Controlling for general cognitive ability, education level, age, and gender, none of the EFs significantly predicted scientific or total creative achievements, $p \mathrm{~s}>.19$.

\subsection{IT versus Artistic professional domains and EFs}

Logistic regression was performed to ascertain the effect of overall EF on the IT and artistic professional occupations. The logistic regression model was significant, $\chi^{2}=5.34, p=.02$. The model 
explained 19\% (Nagelkerker $\mathrm{R}^{2}$ ) of the variance in the IT versus artistic professional domains. There was a sensitivity of $61 \%$ and specificity of $44 \%$ for correctly identifying artistic professional domains (total $53 \%$ of correctly classified cases). Higher overall EF was associated with an increased likelihood of being in the artistic, rather than in the IT, professions, Wald $=4.25, p=.04$, OR $=3.67,95 \% \mathrm{CI}[1.07 ; 12.66]$.

The logistic regression model was not statistically significant controlling for general cognitive ability, education level, age, and gender, $\chi^{2}=8.13, p=.15$. The model explained $30 \%\left(\right.$ Nagelkerker $\left.\mathrm{R}^{2}\right)$ of the variance in the IT versus artistic professional domains. There was a sensitivity of $89 \%$ and specificity of $50 \%$ for correctly identifying artistic professional domains (total $72 \%$ of correctly classified cases). Higher overall EF remained significantly associated with an increased likelihood of being in the artistic, rather than in the IT professions, Wald $=4.07, p=.04, \mathrm{OR}=4.67,95 \% \mathrm{CI}[1.05 ; 20.85]$ (Table 5). General cognitive ability, however, was not associated with being in either profession, either with $($ Wald $=.01, p=.97, \mathrm{OR}=1.00,95 \% \mathrm{CI}[.81 ; 1.23])$, or without $($ Wald $=.01, p=.96, \mathrm{OR}=1.01,95 \% \mathrm{CI}$ $[.82 ; 1.23])$ controlling for the overall EF.

Logistic regression was performed to ascertain the independent effects of Updating, Shifting, and Inhibition on the IT and artistic professions. The logistic regression model was statistically significant, $\chi^{2}$ $=12.14, p=.007$. The model explained $40 \%\left(\right.$ Nagelkerker $\left.\mathrm{R}^{2}\right)$ of the variance in the IT and artistic professions. There was a sensitivity of $78 \%$ and specificity of $63 \%$ for correctly identifying artistic professional domains (total 71\% of correctly classified cases). The model indicated that, controlling for shared variance of the other EFs, Shifting ability was associated with an increased likelihood of being involved in the artistic, rather than in the IT professions, Wald $=3.92, p=.048, \mathrm{OR}=4.10,95 \% \mathrm{CI}[1.02$; $16.56]$.

The logistic regression model was statistically significant with general cognitive ability, education level, age, and gender in the model in addition to the other EFs, $\chi^{2}=18.14, p=.01$. The model explained $58 \%\left(\right.$ Nagelkerker $\mathrm{R}^{2}$ ) of the variance in the IT and artistic professions. There was a sensitivity of $83 \%$ 
and specificity of $71 \%$ for correctly identifying artistic professional domains (total $78 \%$ of correctly classified cases). Better Shifting ability remained significantly associated with an increased likelihood of being involved in the artistic, rather than in the IT professions, Wald $=4.24, p=.04, \mathrm{OR}=31.03,95 \% \mathrm{CI}$ $[1.18 ; 815.52]$. Worse Updating ability was marginally associated with being in the artistic, rather than in the IT professions, Wald $=3.73, p=.053, \mathrm{OR}=.13,95 \% \mathrm{CI}[.02 ; 1.03]$ (Table 5). 


\section{Table 5}

Logistic regressions predicting artistic and IT professional domains.

Model

B $\quad S E \quad$ Wald OR $p \quad 95 \%$ CI

DV: Artistic vs. IT Professional Domains

$\begin{array}{lcccccc}\text { EF total } & 1.54 & 0.76 & 4.07 * & 4.67 & .04 & {[1.05 ; 20.85]} \\ \text { ICAR } & 0.02 & 0.12 & 0.04 & 1.02 & .85 & {[.81 ; 1.26]} \\ \text { Education } & -0.03 & 0.37 & 0.01 & 0.97 & .94 & {[.47 ; 2.01]} \\ \text { Age } & -0.02 & 0.06 & 0.19 & 0.98 & .67 & {[.87 ; 1.09]} \\ \text { Gender } & 1.05 & 0.90 & 1.37 & 2.86 & .24 & {[.49 ; 16.66]}\end{array}$

DV: Artistic vs. IT Professional Domains

$\begin{array}{lllllll}\text { Updating } & -2.06 & 1.07 & 3.73^{\dagger} & 0.13 & .05 & {[.02 ; 1.03]} \\ \text { Shifting } & 3.44 & 1.67 & 4.24^{*} & 31.03 & .04 & {[1.18 ; 815.82]} \\ \text { Inhibition } & .79 & 0.68 & 1.37 & 2.21 & .24 & {[.59 ; 8.31]} \\ \text { ICAR } & 0.38 & 0.21 & 3.34^{\dagger} & 1.46 & .07 & {[.97 ; 2.19]} \\ \text { Education } & 0.05 & 0.43 & 0.01 & 1.05 & .91 & {[.45 ; 2.46]} \\ \text { Age } & 0.04 & 0.07 & 0.37 & 1.04 & .54 & {[.91 ; 1.19]} \\ \text { Gender } & 1.60 & 1.16 & 1.92 & 4.95 & .17 & {[.52 ; 47.57]}\end{array}$

Note. $\quad \mathrm{EF}=$ Executive Function; ICAR = International Cognitive Ability Resource. Positive Betas indicate higher measures for artistic professional domains. 


\section{Discussion}

The purpose of the investigation was to examine the role of EFs in creativity. We examined whether and how Updating, Shifting, and Inhibition, together and uniquely, predict creativity operationalized in three different ways: Performance on a laboratory test of divergent thinking, selfreported creative achievements (arts and science domains), and involvement in artistic (versus IT) professional domains. Results indicated that the role of these EFs differs depending on the measure of creativity. Specifically, fluency of responses on a laboratory test of divergent thinking was predicted by better Updating abilities, controlling for other EFs. People with self-reported artistic achievements were more likely to have better Inhibition abilities, controlling for other EFs. Finally, overall EF, as well as variance unique to Shifting ability, were associated with an increased likelihood of being involved in the artistic rather than IT professions.

\subsection{Divergent thinking and EFS}

Updating abilities were uniquely associated with the fluency of divergent thinking. This result partially replicates previous findings by Benedek and colleagues (2014) showing that divergent thinking, measured with a holistic "creativity" score, was significantly predicted by Updating abilities. Here we report that Updating abilities uniquely predicted fluency, but not originality of divergent thinking. Unlike Benedek and colleagues, we found no association between divergent thinking (fluency or originality) and Inhibition abilities. This could be, at least partially, due to the reliability of the composite variables in our study compared to the reliability in Benedek and colleagues (2014). In their investigation, the EFs were assessed with a single task, while EFs in our study were a combination of the two highest-loading tasks for each EFs. As a consequence, in our study Updating, Shifting, and Inhibition were robustly correlated with one another (Pearson correlations between .44 and .81), while in Benedek and colleagues' study the three EFs were virtually unrelated. Further, divergent thinking was measured differently in the two studies - whereas the average 'creativity' ratings for the top three ideas judged by the independent raters constituted creativity scores in Benedek and colleagues' (2014) investigation, our study examined 
divergent thinking abilities in terms of people's ability to produce a high number of ideas (fluency) versus the uniqueness of their ideas (originality). Additionally, the ATTA task used in this study is comprised of both figural and verbal measures (Goff \& Torrance, 2002), while creativity assessment in Benedek and colleagues' work was only verbal in nature.

Our results indicate that working memory updating plays a prominent role in generating multiple answers (regardless of their novelty) on a divergent thinking task. Given that working memory is strongly linked with both fluid and crystalized intelligence (Ackerman et al., 205; Friedman et al., 2006; Kane, Hambrick, \& Conway, 2005), it is likely that Updating abilities are the common underlying mechanism for the often-debated link between creativity and intelligence (for review, Haier \& Jung, 2008). Indeed, considering the nature of the divergent thinking tests, where participants are required to generate numerous novel uses for common objects (e.g., brick) within a limited amount of time (typically 3-5 minutes), working memory updating is likely to benefit successful performance through retrieval and maintenance/updating of which options have already been used, as well as through the search and retrieval of possible uses from one's memory (cf. Wiley \& Jarosz, 2012). This ability, however, does not appear to influence the originality of responses, as none of the EFs, nor general cognitive ability as assessed with the ICAR measure, were associated with the originality of responses on the ATTA test, indicating that the originality component of creativity may be different from intelligence after all.

\subsection{Real-life creativity and EFs}

Based on the Model of Creativity and Attention (MOCA; Zabelina, 2018), we hypothesized that people in the artistic professional domains would show better overall EF, as well as better Shiftingspecific abilities. These hypotheses were supported - overall EF, as well as variance unique to Shifting, were more likely to be associated with artistic, rather than IT professional domains. These findings are novel in showing that people in the artistic professions have both better overall EFs and better Shiftingspecific abilities. Shifting-specific abilities were previously found to be linked with worse self-restraint in childhood (Friedman et al., 2011). Goeschke (2000), and Herd et al. (2014) also discussed stability and 
flexibility as a tradeoff, suggesting that stable maintenance and flexible switching impose antagonistic constraints on cognitive control processes. It appears that people in the artistic professions may not experience these constraints. People in the artistic professions may be able to actively regulate their thoughts and behavior by guiding their cognition in most appropriate ways, as reflected in their better overall EF, as well as flexibly shift between goals and ideas, as reflected in their better Shifting-specific abilities. The link between Shifting-specific abilities and worse self-restraint in childhood is also in line with prior literature reporting that creativity may be associated with attention-deficit hyperactivity disorder (ADHD; Healey \& Rucklidge, 2006; White \& Shah, 2006), as well as with "leaky" or diffused attention (Zabelina et al., 2015; Zabelina et al., 2016).

Further, people with the higher number of self-reported artistic (but not scientific) creative achievements were more likely to show better Inhibition ability, controlling for other EFs. The role of prepotent response inhibition has previously been discussed in the literature (Edl et al., 2014), and may influence creativity by reducing interference of initial ideas and thus avoiding the tendencies to perseverate on a less creative idea.

Overall, our results are supported by recent neuroimaging studies, which show that creative acts involve a balance in networks between spontaneity (which may relate to flexibility), and top-down control (for reviews, see Beaty, Benedek, Silvia, \& Schacter, 2016; Zabelina \& Andrews-Hanna, 2016). Our findings suggest that involvement in the artistic domains is reflected in both the unity and diversity of EFs - people in the artistic domains can flexibly shift to more original ideas (Shifting-specific), while showing an overall ability to regulate their thoughts and behaviors including overriding dominant responses (overall EF).

\subsection{Limitations and future directions}

Future studies will need to examine the underlying neural mechanisms of these and other EFs in creativity. For example, is the nature of the Shifting association with artistic domains linked with activity in the frontoparietal, default-mode networks, or both? 
In general, the results involving IT and artistic professions should be interpreted with caution, as unequal groups and smaller samples are likely to overestimate the effect sizes. Overall, the sample size is small considering the number of predictors in our regression analyses; thus, future research should replicate and extend these findings with larger sample sizes. Further research may aim at recruiting participants from other professional domains, as well as investigating creativity within each domain separately. For example, there may be quite a bit of variance in the artistic group, as creativity involved in being a photographer may be different than creativity involved in being a writer. It would be important, therefore, to examine the role of EFs in separate creative domains. Additionally, competence within each domain should be taken into account, as the level of competence could contribute to the variance within each group. Finally, success in the professions as a grouping variable could also aid in understanding the role of EFs by reducing variance within each individual group.

Developmental and longitudinal studies are needed to further examine whether people with better Shifting-specific abilities or worse self-restraint are indeed more likely to have artistic abilities, or whether they are more likely to become drawn to the artistic professions because these abilities may be particularly advantageous in artistic professions.

Results involving divergent thinking and creative achievement were correlational in nature, and future experimental studies are needed to examine whether Updating, Shifting, or Inhibition abilities may lead to better performance on the divergent thinking tests, or an increased number of creative achievements over time. Finally, the study involved a high number of tests, thus results should be replicated and interpreted with caution.

\subsection{General conclusions}

This study provides support for the role of EF unity and diversity in creativity, depending on its operational definition. The present findings demonstrate that the fluency of divergent thinking is linked with better working memory updating abilities, which may benefit successful performance through the search and retrieval of possible uses from one's memory. The originality of the responses does not appear 
to be linked with any of the EFs, suggesting that the originality of divergent thinking may be different from intelligence after all. Finally, people in the artistic domains exhibit characteristics of both stability and flexibility, as they are able to flexibly shift mental sets (Shifting), while also exhibiting the propensity to successfully regulate their thoughts and behaviors (Common EF). These results further clarify the Model of Creativity and Attention (Zabelina, 2018) by indicating which specific EFs are most relevant for which features or operational definitions of creativity. 


\section{References}

Ackerman, P. L., Beier, M. E., \& Boyle, M. O. (2005). Working memory and intelligence: The same or different constructs? Psychological Bulletin, 131, 30-60.

Andrews-Hanna, J. R., Reidler, J. S., Sepulcre, J., Poulin, R., \& Buckner, R. L. (2010). Functionalanatomic fractionation of the brain's default network. Neuron, 65, 550-62.

Batey, M., \& Furnham, A. (2006). Creativity, intelligence, and personality: A critical review of the scattered literature. Genetic, Social, and General Psychology Monographs, 132(4), 355-429.

Beaty, R. E., Benedek, M., Silvia, P. J., \& Schacter, D. L. (2016). Creative cognition and brain network dynamics. Trends in Cognitive Sciences, 20(2), 87-95.

Benedek, M., Franz, F., Heene, M., \& Neubauer, A. C. (2012). Differential effects of cognitive inhibition and intelligence on creativity. Personality and Individual Differences, 53, 480-485.

Benedek, M., Jauk, E., Sommer, M., Arendasy, M., \& Neubauer, A. C. (2014). Intelligence, creativity, and cognitive control: The common and differential involvement of executive functions in intelligence and creativity. Intelligence, 46, 73-83.

Buckner, R. L., Andrews-Hanna, J. R., \& Schacter, D. L. (2008). The brain's default network: Anatomy, function, and relevance to disease. Annals of the New York Academy of Sciences, 1124, 1-38.

Carson, S. H., Peterson, J. B., \& Higgins, D. M. (2003). Decreased latent inhibition is associated with increased creative achievement in high-functioning individuals. Journal of Personality and Social Psychology, 85, 499-506.

Carson, S. H., Peterson, J. B., \& Higgins, D. M. (2005). Reliability, validity, and factor structure of the creative achievement questionnaire. Creativity Research Journal, 17(1), 37-50.

Cheng, L., Hu, W., Jia, X., \& Runco, M. A. (2016). The different role of cognitive inhibition in early versus late creative problem finding. Psychology of Aesthetics, Creativity, and the Arts, 10(1), 32. 
Christoff, K., Irving, Z. C., Fox, K. C. R., Spreng, R. N., \& Andrews-Hanna, J. R. (2016). Mindwandering as spontaneous thought: a dynamic framework. Nature Reviews Neuroscience, 17, $718-731$.

Chrysikou, E. G., \& Thompson- Schill, S. L. (2011). Dissociable brain states linked to common and creative object use. Human Brain Mapping, 32, 665 - 675.

Cohen, J. (1992). A power primer. Psychological Bulletin, 112, 155-159.

Condon, D. M., \& Revelle, W. (2014). The International Cognitive Ability Resource: Development and initial validation of a public-domain measure. Intelligence, 43, 52-64.

De Dreu, C. K. W., Nijstad, B. A., Baas, M., Wolsink, I., \& Roskes, M. (2012). Working memory benefits creative insight, musical improvisation, and original ideation through maintained taskfocused attention. Personality and Social Psychology Bulletin, 38, 656-669.

Edl, S., Benedek, M., Papousek, I., Weiss, E. M., \& Fink, A. (2014). Creativity and the Stroop interference effect. Personality and Individual Differences, 69, 38-42.

Ellamil, M., Dobson, C., Beeman, M., \& Christoff, K. (2011). Evaluative and generative modes of thought during the creative process. NeuroImage, 59, 1783-1794.

Eysenck, H. J. (1995). Genius. The natural history of creativity. Cambridge, UK: Cambridge University Press.

Feist, G. J. (1998). A meta-analysis of personality in scientific and artistic creativity. Personality and Social Psychology Review, 2(4), 290-309.

Fleming, K. A., Heintzelman, S. J., \& Bartholow, B. D. (2016). Specifying associations between conscientiousness and executive functioning: Mental set shifting, not prepotent response inhibition or working memory updating. Journal of Personality, 84, 348-360.

Friedman, N. P., \& Miyake, A. (2017). Unity and diversity of executive functions: Individual differences as a window on cognitive structure. Cortex, 86, 186-204. 
Friedman, N. P., Miyake, A., Altamirano, L. J., Corley, R. P., Young, S. E., Rhea, S.-A., \& Hewitt, J. K. (2016). Stability and change in executive function abilities from late adolescence to early adulthood: A longitudinal twin study. Developmental Psychology, 52, 326-340.

Friedman, N. P., Miyake, A., Corley, R. P., Young, S. E., DeFries, J. C., \& Hewitt, J. K. (2006). Not all executive functions are related to intelligence. Psychological Science, 17, 172-179.

Friedman, N. P., Miyake, A., Young, S. E., DeFries, J. C., Corley, R. P., \& Hewitt, J. K. (2008). Individual differences in executive functions are almost entirely genetic in origin. Journal of Experimental Psychology. General, 137, 201-25.

Friedman, N. P., Miyake, A., Robinson, J. L., \& Hewitt, J. K. (2011). Developmental trajectories in toddlers' self-restraint predict individual differences in executive functions 14 years later: A behavioral genetic analysis. Developmental Psychology, 47, 1410-1430.

Cheng, L., Hu, W., Jia, X., \& Runco, M. A. (2016). The different role of cognitive inhibition in early versus late creative problem finding. Psychology of Aesthetics, Creativity, and the Arts, 10(1), 32.

Goff, K. \& Torrance, E. P. (2002). Abbreviated Torrance Test for Adults. Bensenville, IL: Scholastic Testing Service.

Goschke, T. (2000). Intentional reconfiguration and involuntary persistence in task set switching. In S. Monsell \& J. Driver (Eds.), Control of cognitive processes: Attention and performance XVIII (pp. 331-355). Cambridge, MA: MIT Press.

Groborz, M., \& Necka, E. (2003). Creativity and cognitive control: Explorations of generation and evaluation skills. Creativity Research Journal, 15(2-3), 183-197.

Gusnard, D. A., \& Raichle, M. E. (2001). Searching for a baseline: functional imaging and the resting human brain. Nature Reviews Neuroscience, 2(10), 685.

Haier, R. J., \& Jung, R. E. (2008). Brain imaging studies of intelligence and creativity: What is the picture for education? Roeper Review, 30(3), 171-180. 
Healey, D. M., \& Rucklidge, J. J. (2008). The relationship between ADHD and creativity. ADHD Report, $16,1-5$.

Herd, S. A., O'Reilly, R. C., Hazy, T. E., Chatham, C. H., Brant, A. M., \& Friedman, N. P. (2014). A neural network model of individual differences in task switching abilities. Neuropsychologia, 62, $375-389$.

Ito, T. A., Friedman, N. P., Bartholow, B. D., Correll, J., Loersch, C., Altamirano, L. J., \& Miyake, A. (2015). Toward a comprehensive understanding of executive cognitive function in implicit racial bias. Journal of Personality and Social Psychology, 108, 187-218.

Jauk, E., Benedek, M., Dunst, B., \& Neubauer, A. C. (2013). The relationship between intelligence and creativity: New support for the threshold hypothesis by means of empirical breakpoint detection. Intelligence, 41, 212-221.

Kane, M. J., Hambrick, D. Z., \& Conway, D. Z. (2005). Working memory capacity and fluid intelligence are strongly related constructs: Comment on Ackerman, Beier, and Boyle (2005). Psychological Bulletin, 131, 66-71.

Kim, K. H., Cramond, B., \& VanTassel-Baska, J. (2010). The relationship between creativity and intelligence. The Cambridge handbook of creativity, 395-412.

Kozbelt, A. (2008). Hierarchical liner modeling of creative artists' problem solving behaviors. Journal of Creative Behavior, 42, $181-200$.

Liu, S., Erkkinen, M. G., Healey, M. L., Xu, Y., Swett, K. E., Chow, H. M., \& Braun, A. R. (2015). Brain activity and connectivity during poetry composition: Toward a multidimensional model of the creative process. Human Brain Mapping, 36, 3351-3372.

Martindale, C. (1999). Biological bases of creativity. In R. J. Sternberg (Ed.), Handbook of creativity (pp. 137-152). Cambridge, U.K.: Cambridge University Press.

Miyake, A., Emerson, M. J., \& Friedman, N. P. (2000). Assessment of executive functions in clinical settings: Problems and recommendations. Seminars in Speech and Language, 21, 169-183. 
Miyake, A., \& Friedman, N. P. (2012). The nature and organization of individual differences in executive functions: Four general conclusions. Current Directions in Psychological Science, 21, 8-14.

Miyake, A., Friedman, N. P., Emerson, M. J., Witzki, A. H., \& Howerter, A. (2000). The unity and diversity of executive functions and their contributions to complex "frontal lobe" tasks: A latent variable analysis. Cognitive Psychology, 41, 49-100.

Nusbaum, E. C., \& Silvia, P. J. (2011). Are intelligence and creativity really so different?: Fluid intelligence, executive processes, and strategy use in divergent thinking. Intelligence, 39(1), 3645.

Rabbitt, P. (1997b). Introduction: Methodologies and models in the study of executive function. In P. Rabbitt (Ed.), Methodology of frontal and executive function (pp. 1-38). Hove, UK: Psychology Press.

Runco, M. A., \& Jaeger, G. J. (2012). The standard definition of creativity. Creativity Research Journal, 24, 92-96.

Silvia, P. J. (2008). Another look at creativity and intelligence: Exploring higher-order models and probable confounds. Personality and Individual Differences, 44, 1012-1021.

Silvia, P. J., Winterstein, B. B., Willse, J. T., Barona, C. M., Cram, J. T., Hess, K. I., et al. (2008). Assessing creativity with divergent thinking tasks: Exploring the reliability and validity of new subjective scoring methods. Psychology of Aesthetics, Creativity, and the Arts, 2, 68-85.

Takeuchi, H., Taki, Y., Hashizume, H., Sassa, Y., Nagase, T., Nouchi, R., \& Kawashima, R. (2011). Failing to deactivate: The association between brain activity during a working memory task and creativity. NeuroImage, 55, $681-687$.

Thompson-Schill, S. L., Ramscar, M., \& Chrysikou, E. G. (2009). Cognition without control: When a little frontal lobe goes a long way. Current Directions in Psychological Science, 18(5), 259-263.

Torrance, E. P. (1974). The Torrance Tests of Creative Thinking-Norms - Technical manual research edition, figural tests, Forms $A$ and B. Princeton, NJ: Personnel Press. 
Wallach, M. A., \& Kogan, N. (1965). Modes of thinking in young children: A study of the creativityintelligence distinction. New York, NY: Holt, Rinehart, \& Winston.

White, H. A., \& Shah, P. (2006). Uninhibited imaginations: creativity in adults with attentiondeficit/hyperactivity disorder. Personality and Individual Differences, 40(6), 1121-1131.

Wiley, J., \& Jarosz, A. F. (2012). Working memory capacity, attentional focus, and problem solving. Current Directions in Psychological Science, 21, 258-262.

Zabelina, D. L. (in press). Attention and creativity. In R. E. Jung \& O. Vartanian (Eds.), The Cambridge Handbook of the Neuroscience of Creativity. Cambridge, UK: Cambridge University Press.

Zabelina, D. L., \& Andrews-Hanna, J. R. (2016). Dynamic network interactions supporting internallyoriented cognition. Current Opinion in Neurobiology, 40, 86-93.

Zabelina, D. L., O’Leary, D., Pornpattananangkul, N., Nusslock, R., \& Beeman, M. (2015). Creativity and sensory gating indexed by the P50: Selective versus leaky sensory gating in divergent thinkers and creative achievers. Neuropsychologia, 69, $77-84$.

Zabelina, D. L., \& Robinson, M. D. (2010). Creativity as flexible cognitive control. Psychology of Aesthetics, Creativity, and the Arts, 4, 136 - 143.

Zabelina, D. L., Robinson, M. D., Council, J. R., \& Bresin, K. (2012). Patterning and nonpatterning in creative cognition: Insights from performance in a random number generation task. Psychology of Aesthetics, Creativity, and the Arts, 6, 137-145.

Zabelina, D. L., Saporta, A., \& Beeman, M. (2016). Flexible or leaky attention in creative people? Distinct patterns of attention for different types of creative thinking. Memory \& Cognition, 44, 488 $-498$. 